\section{Popularising black holes}

\section{Roman Znajek}

The Collapsing Universe: The Story of Black Holes. By Isaac Asimov. Pp. 204. (Walker: New York; Hutchinson: London and Sydney, 1977.) £4.50.

POPULAR books on black holes seem to consist of either metaphysics or science fiction. It is pleasant to discover that Isaac Asimov's The Collapsing Universe is mainly about astronomy. It is perhaps a bit surprising as well, because Asimov is of course a self-confessed "science-fiction writer of some repute". His book is an attempt to convey non-trivial information about black holes to a readership whose previous knowledge does not extend beyond an ability to multiply. Writing as someone whose social life is occasionally blighted by requests to explain what it is 1 do, I can only state that such a book is highly desirable.

Before a layman can appreciate what black holes are and how they fit into the general scheme of things, he must first Icarn some basic facts concerning the objects to be found in the Universe and the forces that act between them. This takes time, and it is therefore quite proper that most of Asimov's book is not about black holes at all. In chapter 1 Asimov describes the four forces that govern the Universe. Of these four, only gravity and electromagnetism are important over distances larger than the diameter of an atomic nucleus. Asimov carefully explains the inverse-square law of force in a manner that should be comprehensible to a pupil considered incapable of taking ordinary level school physics. An advanced level school student would be in danger of getting seriously bored. Electromagnetism is intrinsically stronger than gravity, and it is electromagnetism that controls virtually all terrestrial phenomena. (Gravity only holds things down.) But gravity does not have the self-cancelling effect inherent in electromagnetism; gravity finds it easier to act over large distances, and it is therefore gravity that determines the motions of planetary systems and galaxies.

Chapter 2 is about the planets. Their masses, escape velocities, densities and other properties are described in considerable (though elementary) detail. Chapter 3, on compressed matter, explains why the planets do not simply collapse under their own weight. Electromagnetic forces between adjacent atoms are strong enough to overcome the total gravitational force acting between all the atoms in the planet. The two forces are at an "eternal standoff". This is not the case with stars; but stars are hot, and it is their heat which results in an outward pressure that balances gravity. There is indeed a standoff, but it is only temporary. Stars radiate their heat away, and must burn nuclear fuel to keep going. The fuel supply is finite, and sooner or later the stars will cool and contract. Even then, total gravitational collapse can be halted by the degeneracy pressure of electrons and neutrons, which does not depend on heat. The resulting objects are white dwarves and neutron stars, and these are described in chapters 4 and 6 respectively. The intermediate chapter links the big bang, stellar evolution and supernovae under the conveniently general heading of "Exploding Matter".

So far, so good. The reader has been given an elementary introduction to a great deal of astronomy. Asimov is clear and usually accurate. (He does seem to be somewhat confused about the relationship between ionisation and degeneracy.) Rather than just describe what astronomers have concluded, he outlines the way in which they have arrived at those conclusions.

This is after all a book on black holes, and they are the subject of the final two chapters. Asimov's treatment of black holes is disappointing. He states that their escape velocity is equal to the speed of light and that "it so happens that physicists are quite certain that no physical object possessing mass can move at a speed equal to or greater than light". This is uncharacteristically dogmatic. A spacetime diagram displaying the motion of light rays in and around a black hole would explain what he means by the escape velocity being the speed of light, and would not demand any more of his readers. Even better, a chapter containing a qualitative description of special relativity would explain why physicists can be so certain that nothing moves faster than light, and would be very worthwhile for its own sake. There are in fact no illusto ations in Asimov's book, and these would surely be a great help.

The basic trouble seems to be that Asimov is stronger on observations than on theory. Black holes are still a rather theoretical subject. His account of the discovery of the X-ray source Cygnus X-1 is fine, and so is his description of the reasoning that leads many astronomers to believe that Cygnus $\mathrm{X}-1$ contains a black hole. What Asimov does not do well is to explain the accretion disk mechanism that is supposed to be responsible for generating the $\mathrm{X}$ rays. He says that particles spiralling into the hole lose gravitational energy, but nowhere does he try to explain what gravitational energy is. One or two down-to-earth examples, such as a stone falling into a well, would help. The vital role of differential rotation and friction in generating the heat that produces the $\mathrm{X}$ rays is not mentioned.

Asimov presents a thoroughly oversimplified version of the Penrose process. With an additional half-page of text he could have got it right. He seems to think that the Penrose process is controversial. Indeed, he goes so far as to state that "almost anything some astronomers suggest about a black hole is denied by other astronomers". This is probably correct in so far as he refers to theories concerning the presence of black holes in specific objects such as quasars or radio galaxies. The general properties of black holes are, however, the province of mathematics rather than physics. Given the fundamental axiom that spacetime obeys the laws of general relativity (and if you are going to write a book about black holes you should at least have some faith in the fundamental axiom) then the properties of black holes are described by mathematical theorems. The Penrose process is such a theorem. Another theorem (which requires one to accept quantum field theory as well as general relativity) is the Hawking effect whereby small black holes emit thermal radiation and evaporate. This generally accepted result is probably the most interesting and significant piece of work carried out on black holes in the past few years. According to Asimov, it is a speculative suggestion, and he only gives it two or three lines. The unimportant and probably incorrect idea that the great Siberian explosion of 1908 was due to a mini-black hole passing through the Earth is given a whole page.

The usual trouble with popularisations is that they treat speculation as established knowledge. The Collapsing Universe is quite exceptional in having the opposite tendency. Asimov is clearly acting from the best of motives. He cannot be accused of sensationalism, although whoever wrote the blurb on the dust-jacket can. Asimov is aware of many of the developments that have taken place in the past few years, but he seems to have little idea of their relative importance. This could be due to haphazard communication between writers like him and the mainstream scientific community, at least as far as black holes are concerned. One possible remedy might be to hold meetings between representative groups of scientists and the writers and journalists whose job it is to present science to the general public.

To sum up, Asimov has written half of a good book on black holes. He ought to have the opportunity of writing the other half, and I look forward to seeing a second edition.

Roman Znajek is an SRC Post-Doctoral Fellow at the Institute of Astronomy, Cambridge, UK. 